\title{
Essen stroke risk score, cerebral cholinergic pathways and cognitive performances in patients with cerebral small vessel disease
}

\author{
Zhu ZHU, Xu HAN, Kun FANG, Yi DONG, Xiang HAN, Qiang DONG* \\ Department of Neurology, Huashan Hospital, State Key Laboratory of Medical Neurobiology, FudanUniversity, China
}

\begin{abstract}
Background: Essen stroke risk score (ESRS) has been proposed for predicting recurrent risk of stroke caused by extra- and intra-cranial atherosclerotic disease. Nevertheless, the role of ESRS in cognition associated with cerebral small vessel disease (CSVD) has not been reported to our knowledge. We investigated the relationship between higher ESRS and impaired cognitive performances in patients with CSVD, with the hypothesis that cerebral cholinergic deafferentation may be one of the mechanisms.

Methods: ESRS and Montreal cognitive assessment (MoCA) were assessed in 102 consecutive patients (mean age (67.2 \pm 8.3 ) years, 48-85 years) with magnetic resonance imaging (MRI) finding of CSVD admitted to our department from 2009 to 2012. The severity of brain cholinergic tracts lesions was measured by the cholinergic pathways hyperintensities scale (CHIPS) score on fluid attenuated inversion recovery (FLAIR) MRI image. Descriptive analysis, pearson correlation as well as stepwise multiple regression analysis was performed.

Results: Of the 102 patients included, for each point increase in ESRS and CHIPS scores, the probability of lower MoCA score increased by 0.696 ( $\mathrm{p}=0.075$ ) and 0.071 ( $\mathrm{p}=0.007$ ) respectively after controlling educational level as a covariate. In the full model, educational level explained $13 \%$ of MoCA variance, while ESRS and CHIPS explained an additional $12 \%$. What is more, higher ESRS resulted in lower MoCA score partially via increase in CHIPS score (0.290 point in MoCA for each point in CHIPS).

Conclusions: Cognitive performance negatively correlated with ESRS in patients with CSVD probably via cerebral cholinergic pathways to some extent. ESRS has potential to be a simple tool for CSVD indicating impaired cerebral cholinergic system
\end{abstract}

\section{Introduction}

Essen stroke risk score (ESRS) has been proposed for stratifying recurrent risk of stroke caused by extra- and intra-cranial atherosclerotic disease [1]. Meanwhile, risk factors of atherosclerotic diseaseare largely shared by cerebral small vessel disease (CSVD) as well $[2,3]$. Visualized as white matter lesions (WMLs), lacunar infarcts, orcerebral microbleeds (CMBs) on magnetic resonance imaging (MRI), CSVD has been found closely related to cognitive impairment [4], one explanation for which is disruption of cholinergic fibers by strategically located white matter hyperintensities [5]. Nevertheless, the specific relationship between the vascular risk factors, CSVD associated cognitive decline and its underlying mechanisms is lacking.

The aim of this study was to investigate the relationship between vascular risk factors evaluated by ESRS and cognitive performances in patients with CSVD, with the hypothesis that cerebral cholinergic deafferentation served, at least partially, as the mechanism of declined cognition.

\section{Patients and methods}

\section{Subjects}

Between January 2009 and December 2012, consecutive patients with MRI finding of CSVD admitted to the department of Neurology, Huashan Hospital, Fudan University were recruited. The assessment process included detailed medical history and physical examination using a standardized protocol, cognitive assessment using the Montreal Cognitive Assessment (MoCA) [6], standard laboratory dementia screen (full blood count, blood biochemistry, thyroid function tests, blood glucose, vitamin B12 and folate levels), brain MRI and CT angiography and/or ultrasound. This study was approved by the Institutional Review Board of Huashan Hospital, Fudan University, and written informed consent was obtained from all participants.

Patients with concomitant diseases which may cause WMLs and cognitive impairment (vitaminB12 deficiency, hypothyroidism, CNS infectious diseases, multiple sclerosis, dementia caused by degenerative diseases, etc)and severe carotid/vertebral artery stenosiswerefurther excluded. The final study sample consisted of a total of 102 patients with CSVD.

Correspondence to: Qiang DONG, MD, PhD, No.12 Middle Urumqi Road, Shanghai, China, 200040, Tel: 021-52887145, E-mail: qiang_dong163@126.com

Xiang HAN, MD, PhD, No.12 Middle Urumqi Road, Shanghai, China, 200040, E-mail: hansletter@163.com

Key words: essen stroke risk score, cerebral cholinergic pathways, cognition, cerebral small vessel disease

Received: January 07, 2016; Accepted: January 19, 2016; Published: January 23, 2016 


\section{Demographic data and risk factors}

Demographic characteristics and presence of vascular risk factors were assessed.ESRS was calculated for each patient with 1 point given for hypertension, diabetes, previous myocardial infarction (MI), other cardiovascular disease (except MI and atrial fibrillation), peripheral arterial disease, previous transient ischemic attack (TIA) or ischemic stroke, age $65-75$ or smoking, and 2 points for age $>75$ [1].

\section{Cognitive assessment}

Cognitive assessment was administered to each patient using MoCA by a trained physician. The MoCA is a one-page, 30-point test that usually takes 15 minutes or less to conduct and includes six cognitive domains: visuospatial/executive functions, naming, attention, abstraction, recall and orientation [6].

\section{Brain MRI and Cholinergic Pathways Hyperintensities Scale (CHIPS)}

All subjects underwent brain MRI using a 3.0-tesla scanner with a standard head coil. T1-weighed, T2-wighte, diffusion weighted and FLAIR (TR/TE_9,002/56 msec effectively, inversion time_2,200 msec, NEX_1, slice thickness_5 mm) imaging were obtained. The presence of white matter signal hyperintensities on the FLAIR images was scored using the cholinergic pathways hyperintensities scale (CHIPS) developed by Bocti et al. [7].This scale provides regionally weighted scores depending on presence of WMH in anatomic landmarks in proximity of the cholinergic medial and lateral pathways, which were separated into 10 regions on 4 index slices spanning the third and lateral ventricles in the axial plane (Figure 1). Severity of WHLs was visually rated on a 3-point scale for each region (0-normal; 1-less than $50 \%$ of region involved; 2 -more than $50 \%$ of region involved). Each slice was weighted to account for the decreasing concentration of cholinergic fibers as they project up and fan out inthe white matter (maximum weight 4 for slice 1 ; minimal weight 1 for slice 4 ). The maximum score is 50 for each hemisphere, while a total maximum of 100 per scan. All scans were assessed by two neurologists who were blind to the clinical information independently.

A, Low external capsule: anterior (right $=1$, left $=1$, factor $=4$, total $=8$ ); posterior $($ right $=1$, left $=1$, factor $=4$, total $=8)$. B, High external capsule: anterior ( right $=1$, left $=1$, factor $=3$, total $=6$ ), posterior $($ right $=1$, left $=1$, factor $=3$, total $=6$ ); cingulated (right $=0$, left $=0$, factor $=4$, total $=0$ ). C, Corona radiate: anterior (right $=1$, left $=1$, factor $=2$, total $=4$ ); posterior $($ right $=1$, left $=1$, factor $=2$, total $=4$ ); cingulated (right $=0$,
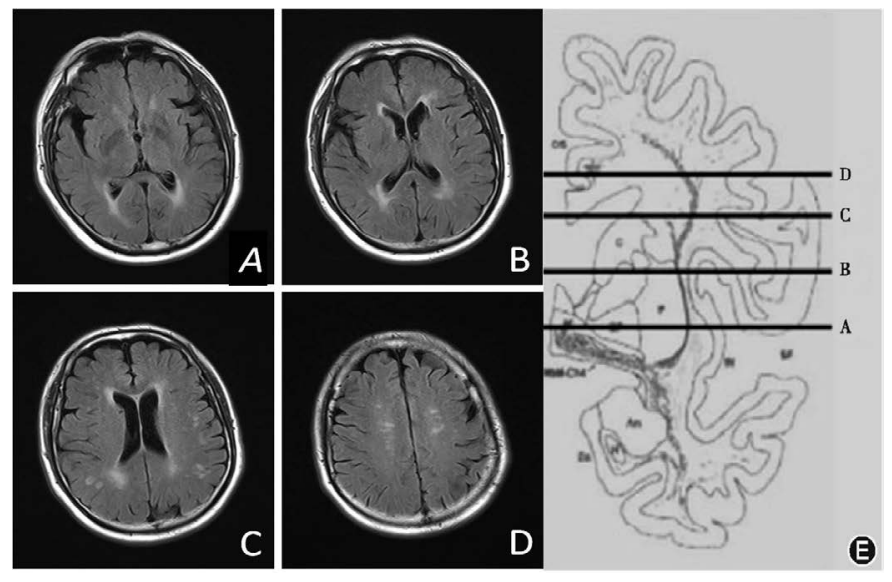

Figure 1. CHIPS scoring system on FLAIR imaging; total score for this subject is 38 .
Table 1. Participant characteristics.

\begin{tabular}{|c|c|}
\hline & $\mathrm{n}=102$ \\
\hline Age, $y^{*}$ & $67.2 \pm 8.3$ \\
\hline Men, \% & $66(64.7)$ \\
\hline \multicolumn{2}{|l|}{ Education } \\
\hline$>12 \mathrm{y}, \%$ & $26(25.5)$ \\
\hline $6-12 y, \%$ & $53(52.0)$ \\
\hline$<6 y, \%$ & $23(22.5)$ \\
\hline Hypertension, \% & $74(72.5)$ \\
\hline Diabetes mellitus, \% & $29(28.4)$ \\
\hline Previous myocardial infarction (MI), \% & $9(8.8)$ \\
\hline Other cardiovascular disease, $\%$ & $2(2.0)$ \\
\hline Peripheral arterial disease, $\%$ & $3(2.9)$ \\
\hline Smoker, \% & $30(29.4)$ \\
\hline Previous TIA or ischemic stroke, $\%$ & $66(64.7)$ \\
\hline ESRS & \\
\hline $0, \%$ & $2(2.0)$ \\
\hline $1, \%$ & $14(13.7)$ \\
\hline $2, \%$ & $22(21.6)$ \\
\hline $3, \%$ & $32(31.4)$ \\
\hline $4, \%$ & $26(25.5)$ \\
\hline $5, \%$ & $6(5.9)$ \\
\hline CHIPS & $34.27 \pm 17.9$ \\
\hline MoCA* & $21.74 \pm 5.1$ \\
\hline
\end{tabular}

SD indicates standard deviation.

*Means with SDs

ESRS: Essen Stroke Risk Score; CHIPS: Cholinergic Pathways Hyperintensities Scale; MoCA: Montreal Cognitive Assessment.

left $=0$, factor $=1$, total $=0$ ). $D$, Centrum semiovale: anterior $($ right $=1$, left $=1$, factor $=1$, total $=2)$; posterior $($ right $=0$, left $=0$, factor $=1$, total $=0$ ). E, Immunohistochemical tracings of the cholinergic pathways from Selden et al. [8].

Demographic characteristics, vascular risk factors, ESRS, CHIPS and MoCA scores of participants are shown in Table 1.

\section{Statistical analyses}

The following categorical and quantitative variables were analyzed: age, gender, vascular risk factors (hypertension, diabetes etc.) educational level, ESRS, CHIPS and MoCA scores. Mean values and corresponding standard errors were calculated. Pearson correlation coefficients were used to assess the direction and magnitude of association between variables. Then we used stepwise multiple regression analysis in different exploratory models. Since educational level has been implicated as a predictor of cognitive performances, simple correlation between education and MoCA was assessed to determine if educational level should be controlled as a covariate ineach model. All data analyses were performed using the software program SPSS 19.0.

\section{Results}

Significant correlation was observed forMoCA score with educational level $(\mathrm{r}=0.378, p<0.001)$, which was therefore included as a covariate. 
Table 2. Correlations among ESRS, CHIPS and MoCA (N=102). ${ }^{*} p<0.05 * * p<0.01$

\begin{tabular}{|c|c|c|c|}
\hline & ESRS & CHIPS & MoCA \\
\hline ESRS & & $0.232^{*}$ & $-0.226^{*}$ \\
\hline CHIPS & & & $-0.349^{* *}$ \\
\hline
\end{tabular}

Table 3. Regression Analysis of CHIPS and MoCA after Adjusting Education (N=102).

\begin{tabular}{|c|c|c|c|c|c|c|}
\hline & \multicolumn{3}{|c|}{ CHIPS } & \multicolumn{3}{c|}{ MoCA } \\
\hline Predictors & B & SEB & P & B & SEB & P \\
\hline ESRS & 3.467 & 1.453 & 0.019 & -0.696 & 0.387 & 0.075 \\
\hline CHIPS & & & & -0.071 & 0.026 & 0.007 \\
\hline
\end{tabular}

Table 2 shows Pearson correlations among the ESRS, CHIPS and MoCA scores. All variables are significantly intercorrelated.

Table 3 provides the results of the stepwise regression analyses of the ESRS $\rightarrow$ CHIPS $\rightarrow$ MoCA path model. In all of the analyses, the covariate was entered as the first step.

The results show that for each point increase in ESRS and CHIPS scores, the probability of lower MoCA score increased by $0.696(p=0.075)$ and $0.071(p=0.007)$ respectively after controlling educational level as a covariate. In the full model, educational level explained $13 \%$ of MoCA variance, while ESRS and CHIPS explained an additional $12 \%$ of MoCA variance. What is more, higher ESRS resulted in lower MoCA score partially via increase in CHIPS score (0.290 point in MoCA for each point in CHIPS).

\section{Discussion}

We found the vascular risk factors assessed by ESRS in our study, with or without stroke, increased the risk of cognitive impairment in patients with CSVD. The finding is consistent with studies in different study populations $[9,10]$. This relation, however, was not significant anymore after controlling education as a covariate $(p=0.075)$, suggesting either the sample size was not large enough or educational level played a more important role in determining the cognitive performances in the subjects.

One possible mechanism of cognitive decline in individuals with CSVD is disruption of cholinergic pathways by strategically located WMLs [11]. The cholinergic neurons of the nucleus basalis of Meynert (nbM) provide the principal cholinergic input of the cerebral cortex [12]. A cross-sectional study conducted by Bohnen et al. found that severity of periventricular WMLs is associated with lower cortical cholinergic activity in middle aged and elderly subjects without dementia. In contrast, there was no significant effect of non periventricular WMLs on global cortical cholinergic activity [5]. What's more, Heuvel et al. performed a 3-year follow-up including repeated MRI and cognitive tests in a large group of over 500 older subjects enrolled in a therapeutic trial, finding that the progression of periventricular WML volume was related to decline in mental processing speed [13]. Both support a regionally specific disruption of cholinergic projection fibers by WMLs. Thus, we chose CHIPS to specifically evaluate the degree of WMLs in the cholinergic pathways which are more closely related to cognition compared to total WML burdens. In addition, the cholinergic axons are mostly unmyelinated and may be more vulnerable to aging or vascular risk factors such as hypertension, diabetes etc [8]. Accordingly, the results indicated that cholinergic pathways deafferentation was significantly associated with both cognitive performances and risk factors of vascular disease even after controlling educational level. Further path analysis showed the indirect pathway mediated by CHIPS served a more significant role in the correlation between vascular risk factors and cognitive performances than the direct way did.

Educational level is generally considered to affect the results of cognitive tests directly without evident association between either risk factors of atherosclerotic disease or WMLs. Besides, no significant correlation was found between the educational level of subjects and ESRS or CHIPS in the simple correlation analysis of our study. Therefore education was controlled as a covariate in our study though it explained comparatively large variance of MoCA. While it is worthy of note that some recent studies suggested the link among the socioeconomic factors including educational level, cerebral white matter integrity and risk of vascular diseases especially stroke in later life [14-16]. The underlying mechanisms were assumed that education helped build cortical network and improve cerebral white matter integrity, as well as increase the resistance of central nervous system against impairments. These findings suggest that for our study, educational level might interact with not only cognitive results but cholinergic pathway reservation and vascular risk factors, despite not significant based on the current data.

There are some limitations of this study. Firstly, MoCA rather than comprehensive neuropsychological assessment battery was applied to assess the cognitive performances of each subject because of the clinical practicability and the study's exploratory nature. This may decrease the sensitivity of our study because the probability of finding positive association between WML burdens and cognitive impairment may be influenced by the cognitive domains assessed. In addition, all patients were from one department, which could cause bias. Thus, the results should be explained with caution.

In summary, this study suggests that vascular risk factors assessed by ESRS affect the cognitive performances in patients with CSVD in a negative way, probably via cerebral cholinergic pathways deafferentation. And ESRS has the potential to be used as a simple tool for CSVD indicating impaired cerebral cholinergic system in clinical practice.

\section{Acknowledgements}

We gratefully acknowledge the members of Department of Neurology, Huashan Hospital, Fudan University, China. Tie LV, Department of Radiology, Huashan Hospital, Fudan University, China.

\section{References}

1. Weimar C, Benemann J, Michalski D, Müller M, Luckner K, et al. (2010) Prediction of recurrent stroke and vascular death in patients with transient ischemic attack or nondisabling stroke: a prospective comparison of validated prognostic scores. Stroke 41: 487-493. [Crossref]

2. Poels MM, Zaccai K, Verwoert GC, Vernooij MW, Hofman A, et al. (2012) Arteria stiffness and cerebral small vessel disease: the Rotterdam Scan Study. Stroke 43: 26372642. [Crossref]

3. Benetos A, Waeber B, Izzo J, Mitchell G, Resnick L, et al. (2002) Influence of age, risk factors, and cardiovascular and renal disease on arterial stiffness: clinical applications. Am J Hypertens 15: 1101-1108. [Crossref]

4. van Dijk EJ, Prins ND, Vrooman HA, Hofman A, Koudstaal PJ, et al. (2008) Progression of cerebral small vessel disease in relation to risk factors and cognitive consequences: Rotterdam Scan study. Stroke 39: 2712-2719. [Crossref]

5. Bohnen NI, Müller ML, Kuwabara H, Constantine GM, Studenski SA (2009) Ageassociated leukoaraiosis and cortical cholinergic deafferentation. Neurology 72: 14111416. [Crossref]

6. Nasreddine ZS, Phillips NA, Bédirian V, Charbonneau S, Whitehead V, et al. (2005) 
The Montreal Cognitive Assessment, MoCA: a brief screening tool for mild cognitive impairment. J Am Geriatr Soc 53: 695-699. [Crossref]

7. Bocti C, Swartz RH, Gao FQ, Sahlas DJ, Behl P, et al. (2005) A new visual rating scale to assess strategic white matter hyperintensities within cholinergic pathways in dementia. Stroke 36: 2126-2131. [Crossref]

8. Selden NR, Gitelman DR, Salamon-Murayama N, Parrish TB, Mesulam MM (1998) Trajectories of cholinergic pathways within the cerebral hemispheres of the human brain. Brain 121: 2249-2257. [Crossref]

9. Van Dijk EJ, Breteler MM, Schmidt R, Berger K, Nilsson LG, et al. (2004) The association between blood pressure, hypertension, and cerebral white matter lesions: cardiovascular determinants of dementia study. Hypertension 5: 625-630.

10. Kovacic JC, Fuster V (2012) Atherosclerotic risk factors, vascular cognitive impairment, and Alzheimer disease. Mt Sinai J Med 79: 664-673. [Crossref]

11. Swartz RH, Sahlas DJ, Black SE (2003) Strategic involvement of cholinergic pathways and executive dysfunction: Does location of white matter signal hyperintensities matter? J Stroke Cerebrovasc Dis 12: 29-36. [Crossref]
12. Mesulam MM, Geula C (1988) Nucleus basalis (Ch4) and cortical cholinergic innervation in the human brain: observations based on the distribution of acetylcholinesterase and choline acetyltransferase. J Comp Neurol 275: 216-240. [Crossref]

13. Van den Heuvel DM, ten Dam VH, de Craen AJ, et al. (2006) Increase in periventricular white matter hyperintensities parallels in mental processing speed in a nondemented elderly population. J Neurol Neurosurg Psychiatry 77: 149-153. [Crossref]

14. Plassman BL, Williams JW Jr, Burke JR, Holsinger T, Benjamin S (2010) Systematic review: factors associated with risk for and possible prevention of cognitive decline in later life. Ann Intern Med 153: 182-193. [Crossref]

15. Dehaene S, Pegado F, Braga LW, Ventura P, Nunes Filho G, et al. (2010) How learning to read changes the cortical networks for vision and language. Science 330: 1359-1364. [Crossref]

16. Farfel JM , Nitrini R, Suemoto CK, Grinberg LT, Ferretti RE, et al. (2013) Very low levels of education and cognitive reserve: a clinicopathologic study. Neurology 81: 650657. [Crossref]

Copyright: (C)2016 ZHU Z. This is an open-access article distributed under the terms of the Creative Commons Attribution License, which permits unrestricted use, distribution, and reproduction in any medium, provided the original author and source are credited. 